\title{
Pressure Monitoring using Hybrid fs/ps Rotational CARS
}

\author{
Sean P. Kearney ${ }^{1}$ \\ Sandia National Laboratories, Albuquerque, New Mexico, 87185 \\ Paul M. Danehy ${ }^{2}$ \\ NASA Langley Research Center, Hampton, Virginia, 23681
}

\begin{abstract}
We investigate the feasibility of gas-phase pressure measurements at $\mathrm{kHz}$-rates using fs/ps rotational CARS. Femtosecond pump and Stokes pulses impulsively prepare a rotational Raman coherence, which is then probed by a high-energy 6-ps pulse introduced at a time delay from the Raman preparation. Rotational CARS spectra were recorded in $\mathbf{N}_{2}$ contained in a room-temperature gas cell for pressures from 0.1 to $3 \mathrm{~atm}$ and probe delays ranging from 10-330 ps. Using published self-broadened collisional linewidth data for $\mathrm{N}_{2}$, both the spectrally integrated coherence decay rate and the spectrally resolved decay were investigated as means for detecting pressure. Shot-averaged and single-laser-shot spectra were interrogated for pressure and the accuracy and precision as a function of probe delay and cell pressure are discussed. Single-shot measurement accuracies were within 0.1 to $6.5 \%$ when compared to a transducer values, while the precision was generally between $1 \%$ and $6 \%$ of measured pressure for probe delays of $200 \mathrm{ps}$ or more, and better than $2 \%$ as the delay approached $300 \mathrm{ps}$. A byproduct of the pressure measurement is an independent but simultaneous measurement of the gas temperature.
\end{abstract}

\section{Introduction}

$\mathrm{L}$ ASER diagnostics offer well-documented capability for probing of gas-phase media such as flames and compressible flow fields. A key advantage of these optical techniques is the ability to monitor multiple parameters simultaneously. Coherent anti-Stokes Raman scattering (CARS) is a particularly attractive spectroscopic tool for simultaneous monitoring of temperature and species concentrations in combustion [1-6]. CARS pressure measurements have also been demonstrated in compressible flow fields, but pressure monitoring has received comparatively less attention. The most detailed demonstration of CARS as a pressure diagnostic to date was conducted by Lucht and coworkers, who used dual-pump CARS [7] and high-resolution vibrational CARS [8, 9] of air and $\mathrm{N}_{2}$ in gas cells and underexpanded jets. The measurements showed feasibility of CARS pressure measurements which exploit the sensitivity of Raman linewidths to collisions, and the competing effect of collisional narrowing. These systems utilized nanosecond laser pulses that yield CARS spectra from an essentially steady-state Raman process at a maximum single-shot data rate of $10 \mathrm{~Hz}$. Femtosecond laser pulses have received significant recent attention for gas-phase CARS diagnostics [10-17]. Impulsive preparation of a Raman coherence with these ultrafast broadband pulses with subsequent time-delayed probing enables direct monitoring of collisional processes [18-21], such that "hybrid" systems which exploit both temporal and spectral information for pressure monitoring are now feasible. Commercial femtosecond amplifiers routinely operate at repetition rates of $1 \mathrm{kHz}$ or more, so that dynamics of the pressure field might also be resolved. In this work, we present a feasibility study in which the pressure sensitivity of transient pure-rotational CARS spectra of $\mathrm{N}_{2}$ in a room-temperature cell is investigated. Temporal dynamics of both the temporally resolved CARS spectra and the spectrally integrated decay of the CARS signal are measured and compared with theoretical predictions. CARS-measured pressures are compared to transducer values, and schemes for practical measurement applications are discussed.

\footnotetext{
${ }^{1}$ Principal Member of the Technical Staff, Engineering Sciences Center, PO Box 5800/Mail Stop 0826, spkearn@ sandia.gov, Associate Fellow AIAA

${ }^{2}$ Research Scientist, Associate Fellow AIAA.
} 
In addition to the CARS measurements mentioned above, a few other molecular-spectroscopic-based methods of non-intrusively measuring in-stream static pressure have been demonstrated. However, compared to temperature, velocity and species concentration measurement techniques, there are relatively few methods of measuring pressure optically [22]. Planar laser-induced fluorescence has been used to measure image pressure fields in flows containing trace quantities of $\mathrm{I}_{2}$ [23]; $\mathrm{OH}$ [24]; $\mathrm{NO}$ [25] and several percent levels of $\mathrm{CO}_{2}$ [26]. While most of these fluorescence measurements provide quantitative images, they are mostly time-averaged, and these resonant gases are not generally present in sufficient quanitites in most flows and often cannot be seeded. Rayleigh scattering, which works in unseeded flows, has been used to image pressure [27], but measurements were also time averaged. Time resolved Rayleigh scattering measurements of temperature, velocity and density have been performed at rates up to 32 $\mathrm{kHz}$ [28]. Pressure was not directly measured in these experiments although it could be determined with $3-4 \%$ precision at $1 \mathrm{kHz}$ from directly measured temperature and density, although this approach is susceptible to errors caused by averaging during this measurement interval [29]. Pressure has also been measured in supersonic flows using time-averaged laser-induced thermal acoustics (LITA) spectra, although the measurement volume is relatively large (15 mm long) [30]. In summary, most of these methods are time averaged and those that can provide single shot measurements are generally limited to a few percent precision at best. The proposed method is a single-point measurement having about $1 \mathrm{~mm}$ spatial resolution with an effectively instantatneous ( $<1 \mathrm{~ns})$ measurement time and can acquire data at rates at or above $1 \mathrm{kHz}$.
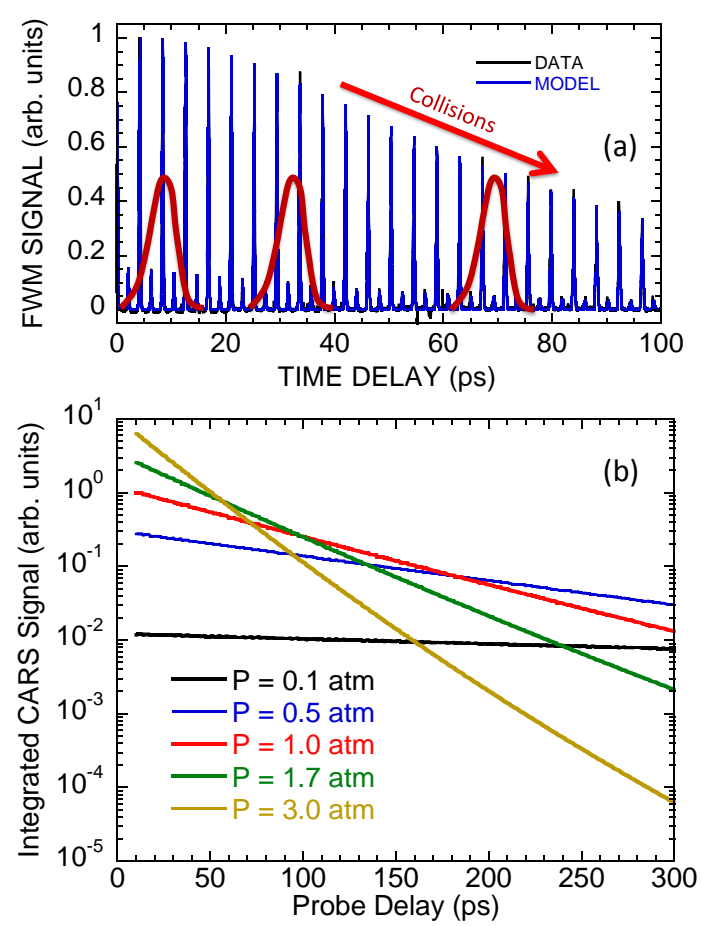

Figure 1. Measured and calculated decay of the rotational coherence in $\mathrm{N}_{2}$ at room temperature and a pressure of 0.82 atm obtained with a 100 fs probe laser, shown with 6 ps probe pulse shapes superposed at three different delays (a). Calculated scaling of the spectrally integrated CARS signal strength at room temperature for a 6-ps duration probe beam at pressures from $0.1-3.0 \mathrm{~atm}(\mathrm{~b})$.

\section{Principle of Hybrid CARS Pressure Measurement}

The pressure sensitivity of the pure-rotational CARS signal arises from collisional dephasing of the coherence that is prepared by broadband, nearly transform-limited pump and Stokes pulses. This impulsive preparation occurs on a time scale of $\sim 100 \mathrm{fs}$, which is much shorter than the 10-100 ps collisional time scales of interest. The measured decay of the room-temperature $\mathrm{N}_{2}$ rotational coherence is shown in Figure 1a. The 100-fs pump and Stokes pulses arrive at time $t=0$ and the transient strength of the induced Raman polarization is monitored by a mechanically scanned 100-fs probe beam. The coherence decay is characterized by "recurrence" peaks that arise due to the approximately even frequency spacing between the rotational Raman transitions [31], which are superposed atop a global exponential decay caused by gas-phase collisions. The coherence is temperature and pressure sensitive, and can be modeled in the time domain as,

$$
\chi(t) \sim P / k T \sum_{\Delta J= \pm 2} \gamma^{2} b_{J} F_{J}\left(N_{J^{\prime}}-N_{J}\right) \exp \left(i \omega_{J} t\right) \exp \left(-P \Gamma_{J} t\right)
$$

In Eq. 1, the factor $P / k T$ is the total number density, where $P$ is the pressure, $T$ is the temperature, and $k$ is the Boltzmann constant. The sum in Eq. 2 is taken over all allowed rotational transitions with a change in rotational quantum number from initial $J$ to final $J^{\prime}=J \pm 2$ states; $\gamma^{2}$ is the anisotropy of the mean polarizability tensor; $b_{J}$ and $F_{J}$ are the Placzek-Teller [32] and Herman-Wallis factors [33], respectively; the $N_{J}$ are the temperature-dependent Boltzmann populations; $\omega_{J}$ are the Raman frequencies; and $\Gamma_{J}$ are the collisional Raman linewidths in $\mathrm{sec}^{-1} / \mathrm{atm}$ [18]. 


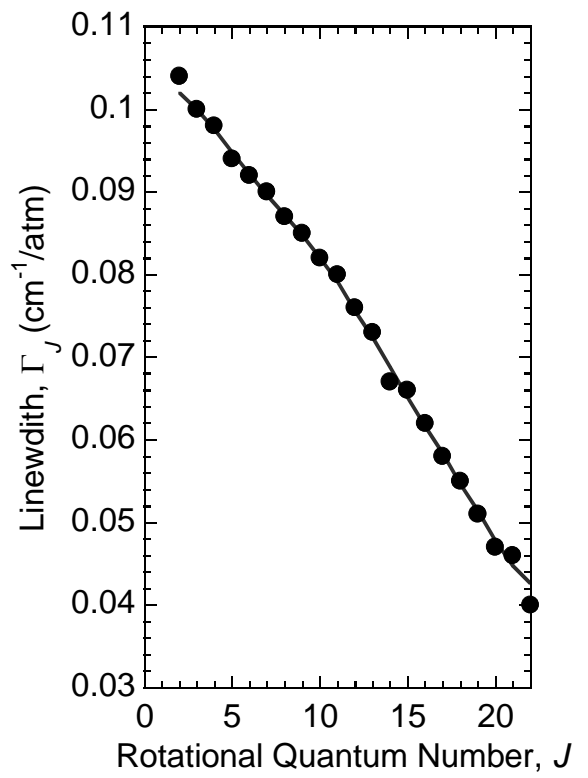

Figure 2. Self-broadened linewidths for the N2 S-branch transitions at room temperature measured by Kliewer et al. [18].

The first-order impact of pressure on $\chi$ is the overall exponential decay, arising from the $\exp \left(-P \Gamma_{J} t\right)$ factor in Eq. 1 . When a ps-duration probe pulse is introduced at time delay, $\tau$, the CARS frequency spectrum is given by the Fourier components of the product of $\chi$ and the probe pulse electric field amplitide,

$$
S(\omega ; \tau)=\left|\int \chi(t) E_{p r}(t-\tau) \exp (-i \omega t) d t\right|^{2} .
$$

When the calculated spectra from Eqs. 1 and 2 are integrated over all $\omega$, this expression yields pressure-dependent decay curves shown in Figure 1b, where the time-constant of the decay near room temperature scales roughly as $\delta \tau_{p}$ $\sim 1 / P$. The decay curves are normalized by an overall scaling factor so that the signal at $1 \mathrm{~atm}$ and a probe delay of $10 \mathrm{ps}$ is equal to unity, while preserving the relative scaling of the curves to illustrate relative signal strengths with pressure. The nominal scaling of the CARS signal strength as $\sim P^{2}$ observed with nanosecond CARS does not occur here, since the relative signal strengths depend strongly on probe delay, as well as the duration of the probe pulse.

The second-order impact of pressure on the measured CARS spectra arises from the $J$ dependence of the collisional linewidths, $\Gamma_{J}$. Room-temperature linewidth measurements compiled by Kliewer et al. [18] are shown in Figure 2, where the $\Gamma_{J}$ are observed to monotonically decrease with $J$, reflecting an increase in the $J$-dependent coherence lifetime through $\delta \tau_{J}=\left(P / P_{\mathrm{o}}\right) \Gamma_{J}^{-1}$. For $\tau$ of order $\delta \tau_{J}$ or greater, the spectra are impacted by collisions, with the intensity of high- $J$ Raman lines becoming proportionally larger than for low- $J$ transitions. The effect of pressure on calculated spectra at $T=294 \mathrm{~K}$ and $\tau=300 \mathrm{ps}$ is shown in Figure 3, where the peak of the spectral intensity envelope is observed to shift toward higher energy as pressure increases. This shifting causes the spectra to appear as if they originate from higher temperature gases as $\tau$ increases [34], but is entirely an effect of pressure. Schemes based on the introduction of multiple probe beams at different delays can be constructed to isolate the effects of temperature and pressure, thereby monitoring both quantities simultaneously on a single-shot basis. Three such probe-pulse envelopes are shown alongside the measured Raman coherence in Figure 1a. At small delays, $\tau \sim 10 \mathrm{ps,}$ collisions have not yet significantly impacted the Raman spectra, and a temperature measurement can be made without regard for the impact of pressure. Introduction of a second probe at a user-optimized delay yields a second CARS spectrum which can be integrated to provide a measure of the pressure-dependent decay, shown in Figure 1b, or spectrally analyzed at fixed temperature to take advantage of the "spectral heating" effect of Figure 3. A multiprobe fs/ps CARS instrument of this kind has recently been demonstrated by Patterson et al. [35] for single-shot monitoring of flame collisional linewidths. 


\section{III. fs/ps CARS Experimental Facility}

A hybrid fs/ps rotational CARS scheme is briefly summarized here, with a schematic of the CARS instrument shown in Figure 4. A commercial "single-box" Ti:sapphire chirped-pulse amplifier (Spectra Physics "Solstice") provides a 1-kHz train of $800 \mathrm{~nm}, 90-100$ fs pulses with a nominal bandwidth of $190-220 \mathrm{~cm}^{-1}$ (FWHM). Eighty percent of the $3.1 \mathrm{~mJ}$ pulse energy is used to generate a picosecond-duration probe beam using the "Second Harmonic Bandwidth Compression" (SHBC Light Conversion) approach described in [17]. The SHBC process utilizes the technique put forth by Raoult et al. [36] to generate picosecond, frequency-narrow pulses by sumfrequency mixing of two broadband pump beams with phase-conjugate linear chirps. Two initially femtosecond pump beams are formed by evenly splitting the $800-\mathrm{nm}$ input beam. Dispersion is added to each pump by Martinez-type [37] grating pulse stretchers, whose lengths are adjusted so that the resulting linear temporal chirps are equal and opposite. Annihilation of the linear chirps occurs via sumfrequency generation in BBO generates a $400 \mathrm{~nm}$ beam with high, 1-1.4 mJ pulse energies. SHBC probe pulse durations are typically $\sim 5-6 \mathrm{ps}$, with linewidths of 3.5$4.0 \mathrm{~cm}^{-1}$ (FWHM). Probe pulse generation by SHBC provides $\sim 100 \times$ greater pulse energies than earlier schemes for ps probe generation based on filtering of the spectral bandwidth [31, 38], and was originally implemented to generate the high signal levels needed to probe at flame temperatures [17]. SHBC is additionally critical for pressure monitoring to achieve sufficient CARS signal at long probe delays, where significant dephasing of the Raman coherence has occurred, significantly reducing the CARS signal by orders of magnitude (see Fig. 1b).

The remaining $20 \%$ of the compressed $800-\mathrm{nm}$ femtosecond pulse is sent through a time of flight delay and then split 50/50 to form separate pump and Stokes pulses, whose energy is regulated to $\sim 40 \mu \mathrm{J} /$ pulse each by variable attenuation with a half-waveplate and polarizer combination. All three laser beams transit through independent computer-controlled delay lines. A planar BOXCARS scheme with a $f=500-\mathrm{mm}$ beam-crossing lens is employed to generate the CARS signal beam, which is collimated at 2:1 magnification by a $f=1000$-mm singlet lens. Isolation of the signal from the input laser beams is performed using dichroic mirrors for the 800 -nm preparation pulses, while careful placement of apertures is employed to isolate the signal beam from the spectrally proximal 400 -nm probe. The signal is dispersed onto a back-illuminated electron-multiplying CCD camera by a 1-m grating spectrograph with a grating groove density of $1800 \mathrm{~mm}^{-1}$. A 2-pixel horizontal bin was applied during readout of the spectra to increase signal counts, with a final spectral dispersion of $0.98 \mathrm{~cm}^{-1} /$ pixel.

Rotational CARS spectra were acquired from $\mathrm{N}_{2}$ in a room-temperature $(T=294 \mathrm{~K})$ gas cell at five nominal pressure settings between 0.1 and $3.0 \mathrm{~atm}$. Probe-beam delays of 10-330 ps from the preparation pulses were used to investigate the pressure sensitivity of the CARS signal at different stages of the rotational coherence decay. CARS spectra were background subtracted and normalized to nonresonant spectra recorded in argon at zero probe delay to account for the finite spectral bandwidth and non-transform-limited performance of the pump and Stokes pulses. The processed spectra were fit using a phenomenological model based on Eqs. 1 and 2, described in greater detail in $[16,17]$, where pressure was added as an additional fitting parameter.

\section{Results and Discussion}

\subsection{Decay of the Spectrally Integrated CARS signal}

The temporal decay of the rotational CARS signal was measured by scanning the probe delay stage from 10-330 ps at fixed gas-cell pressure and integrating the spectra on the detector for 100 laser shots at each stage position. The CARS spectra were then numerically integrated to construct the decay curves shown in Figure 5, where the measured decays are shown as solid lines and least-squares fits to a single exponential decay, $s=\exp (-t / \delta \tau)$, are 


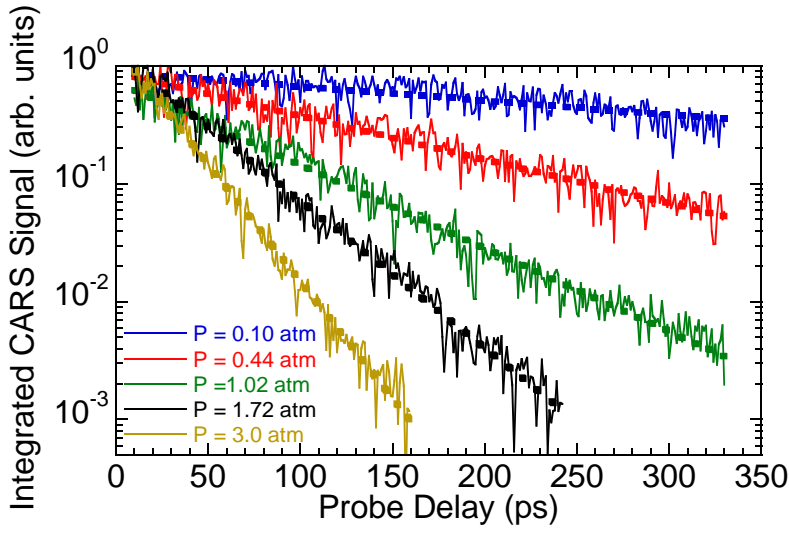

Figure 5. Measured decay of the spectrally integrated CARS signal for $P=0.1-3.0 \mathrm{~atm}$.

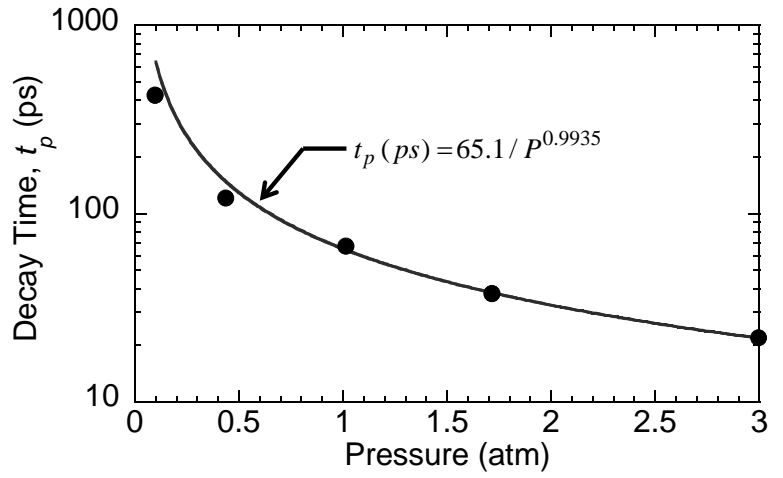

Figure 6. Measured (symbols) and calculated (line) decay times of the spectrally integrated roomtemperature CARS signal strength for $P=0.1-3.0$ atm. The line was not best fit to the measured data.

indicated by the dashed lines. Fitted values of the decay constants, $\delta \tau$, are plotted against the gas cell transducer pressure in Figure 6, where the behavior of $\delta \tau$ predicted by integrating the model spectra from Eqs. 1-2 is shown for comparison. The predicted scaling $\delta \tau \sim P^{-0.9935}$ is just slightly different than the expected $P^{-1}$ behavior. Fitting of the measured $\delta \tau$ yields a weaker pressure scaling of $P^{-0.836}$, that is likely a result of the more rapid decay in the measurements at the two lowest pressures investigated. Increased disagreement at low pressures, where coherence lifetimes are longest, can likely be attributed to small amounts of walk-off in the probe beam, which becomes more important as the probe delay stage is scanned to a large displacement. While, in principle, this observed decay in signal intensity could be used to measure the gas pressure, such a measurement would be sensitive to beam misalignment, resulting in potentially increased random errors in experiments performed in turbulent flows or facilities with excess vibration.

\subsection{Spectrally Resolved Measurements: Shot-Averaged CARS Spectra}

Pressure sensitivity of the measured rotational CARS spectra was investigated by integrating the measured CARS signal for several thousand laser shots for delays of $\tau=11.5100,201,250,300$ and $330 \mathrm{ps}$. Representative shot-averaged spectra and the associated best-fit model results are presented for all five $\mathrm{N}_{2}$ pressures investigated for $\tau=11.5,201$ and $300 \mathrm{ps}$ in Figure 7. At $\tau=11.5 \mathrm{ps}$, the spectral shapes are insensitive to pressure, and the temperature is extracted from the CARS spectra with the pressure fixed at $P=1 \mathrm{~atm}$. Measured temperatures are $\sim 3$ $5 \%$ higher than the known value of $T=294 \mathrm{~K}$ for the present data set. For pressures up to $1 \mathrm{~atm}$, fits to modeled spectra indicate virtually zero collision-induced bias in the measured temperature for $\tau=11.5 \mathrm{ps}$. At $P=1.7$ and 3.0 atm, the pressure-dependent "spectral heating" of the type illustrated in Figure 3 results in an expected temperature bias of $+1 \%$ and +3.1 , respectively, which is consistent with the even higher levels of fitted temperature at these pressures. Previous fs/ps rotational CARS measurements from our lab in room-temperature air [16] at $P=0.82$ atm revealed temperatures that were $\pm 2 \%$ from the known temperature, so that some degree of systematic hightemperature bias exists in the present data. Spectra at probe delays of $\tau=201$ and $300 \mathrm{ps}$ clearly illustrate the collision sensitivity in the measurements, with pressure-dependent changes to the spectral shape most clearly evident at $P=3.0 \mathrm{~atm}$. Spectra at $\tau=201$ and $300 \mathrm{ps}$ were fit by fixing the temperature at the measured value for $\tau=11.5 \mathrm{ps}$ and including the pressure as a variable in the fit. For $P=0.44 \mathrm{~atm}$ and above, fitted pressures from the spectra shown in Figure 7 are within 1.7 to $9.2 \%$ of the value indicated by the gas-cell pressure transducer. At $P=0.1$ atm, the collision time scale is quite long at $\delta \tau=641 \mathrm{ps,} \mathrm{and} \mathrm{the} \mathrm{pressure} \mathrm{sensitivity} \mathrm{at} \mathrm{the} \mathrm{probe} \mathrm{delays} \mathrm{investigated} \mathrm{here}$ is significantly reduced, with increased measurement errors of 26 and $75 \%$ observed in Figure 7. Higher measurement accuracy at this low pressure can likely be obtained by using a longer probe delay, though that was not possible with the existing setup.

The spectral range utilized in fitting was necessarily decreased at the highest pressures and delays shown in Figure 7, where artifacts in the spectra at low Raman shifts are clearly seen in the spectra for $P=1-3$ atm and $\tau=$ 201 and 300 ps. This intense scattering about the Rayleigh line at $\omega=0$ was coherent in nature, and could not be removed from the spectra. The amplitude of this artifact appeared to increase with pressure, and did not possess the same temporal decay as the resonant CARS signal. 


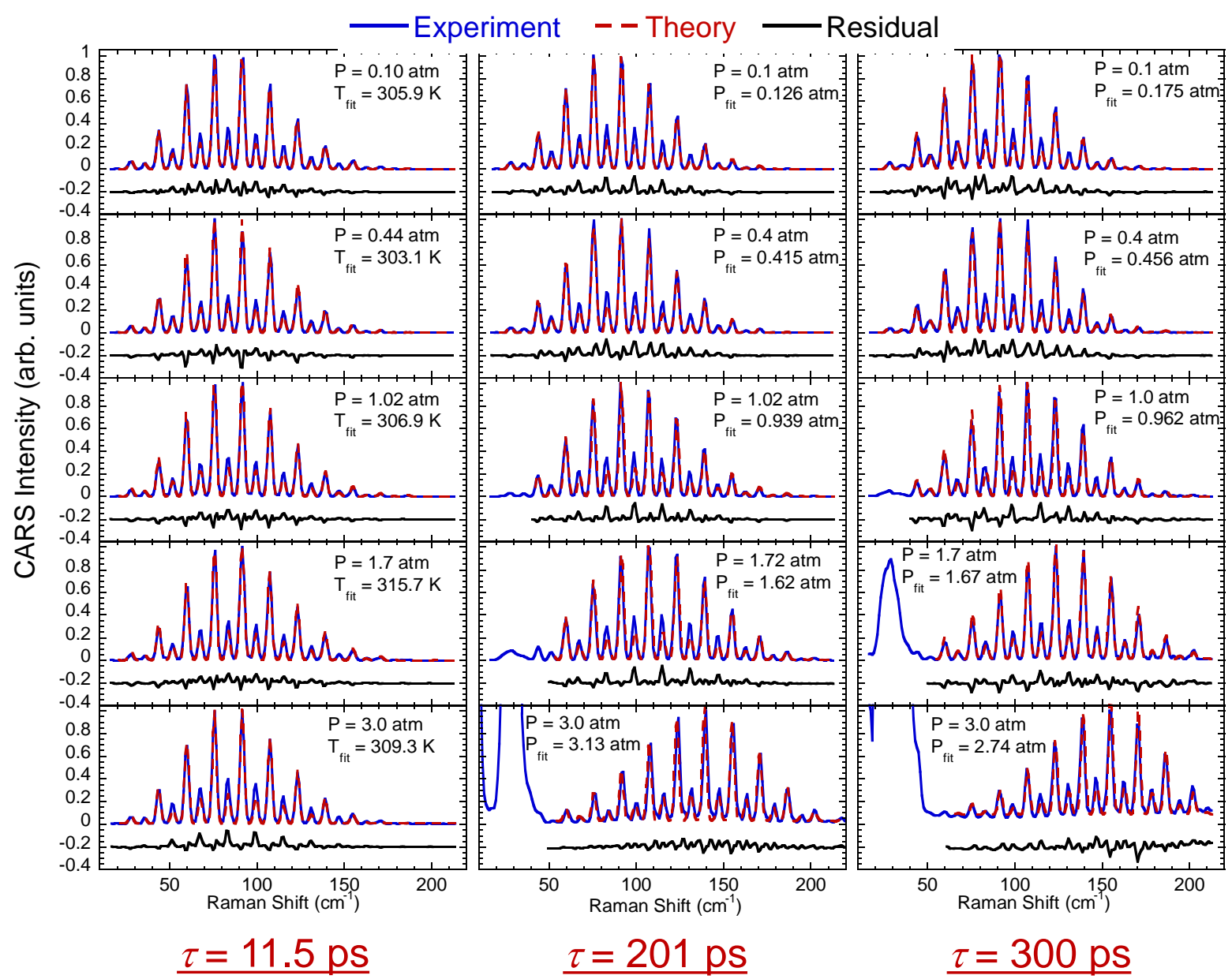

Figure 7. Measured room-temperature rotational fs/ps CARS spectra at a probe delays of $\tau=11.5,201$ and $300 \mathrm{ps}$ for pressures $P=0.1-3.0 \mathrm{~atm}$. The spectra were averaged for 2000 laser shots. 

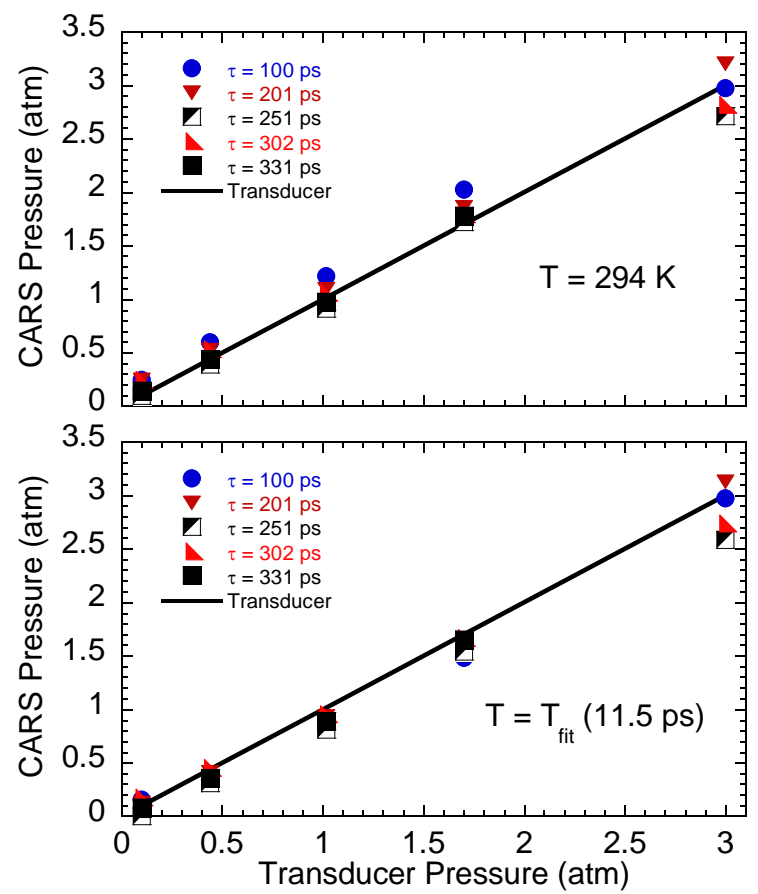

Figure 8. CARS-measured pressures (symbols) plotted against the gas-cell pressure transducer readings.

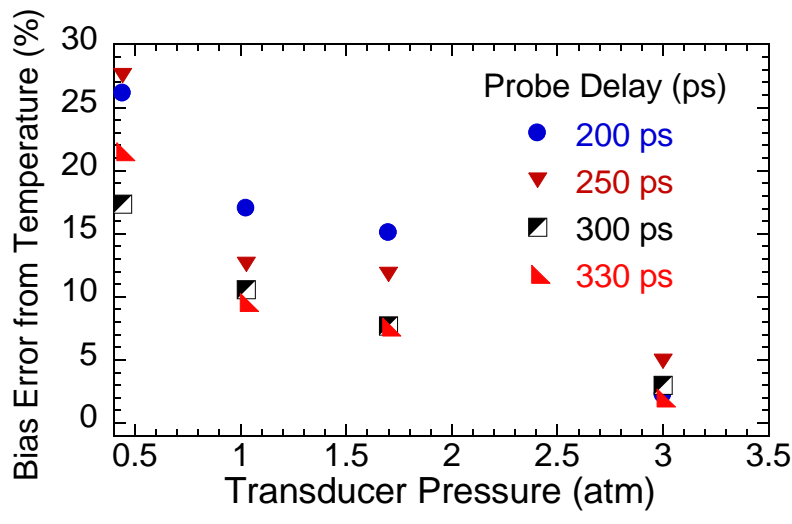

Figure 9. Impact of fitted temperature on CARSmeasured pressure.

The accuracy of the pressure measurements obtained from fitting of 2000-shot-averaged CARS spectra is summarized in Figure 8, where the CARS-measured pressures are plotted against the gas-cell transducer reading for all probe delays. Two sets of results are shown. At the top of the Figure, pressure measurements obtained with the temperature fixed at the known value of $T=294 \mathrm{~K}$ in the spectral fitting are presented. Pressure data obtained by fitting with the temperature fixed at the fitted value at $\tau=11.5 \mathrm{ps}$ are shown in the lower half of the Figure. In both cases, sufficient delay is required to achieve the best measurement accuracy. For $\tau$ $=200 \mathrm{ps}$ and larger, CARS-measured pressures are generally within $10 \%$ of the transducer value for $P=0.44$ to 3.0 atm. The effect of the above-noted 3-5\% high-temperature bias on the fitted pressure is significantly dependent on gas-cell pressure and probe delay, as shown in Figure 9. At $P=3 \mathrm{~atm}$, the temperature effect results in a change of $2-5 \%$ in the fitted pressures, with the error decreasing monotonically with probe delay. The temperature-induced bias then consistently increases as gas-cell pressure is lowered up to worst-case values of $17-27 \%$ at $P=0.44$ atm. This level of potential bias error, particularly at low pressures, illustrates the importance of accurate CARS thermometry.

\subsection{Single-Laser-Shot Pressure Measurements}

Single-laser-shot spectra were additionally acquired at the same nominal pressures and probe delays as the shotaveraged data discussed above. Representative single-shot spectra for $P=0.1-3.0$ atm for a fixed probe delay of $\tau=$ 200 ps are shown along with best-fit model spectra in Figure 10. In this case, the pressure was fit by fixing the temperature at the known value of $T=294 \mathrm{~K}$. At this probe delay, single-shot signal-to-noise is quite good, even at $P=3.0 \mathrm{~atm}$, where the 200-ps delay time is an order of magnitude larger than the measured 1/e coherence decay time of $\delta t=21.6$ ps shown in Figure 6. Singe-shot precision was investigated by constructing histograms of CARSmeasured pressures from ensembles of 2000 spectra, with the results shown in Figure 11. These single-shot results were acquired at a long probe delay of $\tau=298 \mathrm{ps}$ in order to maximize sensitivity at all pressures. The observed measurement precision, as quantified by a single standard deviation in the histogram data is $0.65 \%-1.9 \%$ of the

Table 1. Summary of CARS single-shot pressure measurements.

\begin{tabular}{|c|c|c|c|c|c|}
\hline $\begin{array}{c}P_{\text {cell }} \\
(\mathrm{atm})\end{array}$ & $\begin{array}{c}\bar{P}(\mathrm{~atm}), \sigma_{P} / \bar{P}(\%) \\
\tau=100 \mathrm{ps}\end{array}$ & $\begin{array}{c}\bar{P}(\mathrm{~atm}), \sigma_{P} / \bar{P}(\%) \\
\tau=150 \mathrm{ps}\end{array}$ & $\begin{array}{c}\bar{P}(\mathrm{~atm}), \sigma_{P} / \bar{P}(\%) \\
\tau=200 \mathrm{ps}\end{array}$ & $\begin{array}{c}\bar{P}(\mathrm{~atm}), \sigma_{P} / \bar{P}(\%) \\
\tau=250 \mathrm{ps}\end{array}$ & $\begin{array}{c}\bar{P}(\mathrm{~atm}), \sigma_{P} / \bar{P}(\%) \\
\tau=297-298 \mathrm{ps}\end{array}$ \\
\hline 0.41 & & $0.54,2.9 \%$ & $0.45,2.4 \%$ & $0.59,4.1 \%$ & $0.45,1.9 \%$ \\
\hline 1.07 & & $1.13,1.7 \%$ & $1.05,1.5 \%$ & $1.14,3.1 \%$ & $1.06,0.87 \%$ \\
\hline 1.41 & $1.54,5.9 \%$ & $1.38,4.6 \%$ & $1.44,3.1 \%$ & $1.33,1.8 \%$ & $1.43,1.4 \%$ \\
\hline 2.14 & $1.87,8.3 \%$ & $2.02,6.8 \%$ & $2.04,5.9 \%$ & $2.21,1.6 \%$ & $2.18,0.65 \%$ \\
\hline 3.03 & $2.51,5.8 \%$ & $2.87,2.7 \%$ & $2.98,1.6 \%$ & $3.19,0.8 \%$ & $3.25,1.2 \%$
\end{tabular}




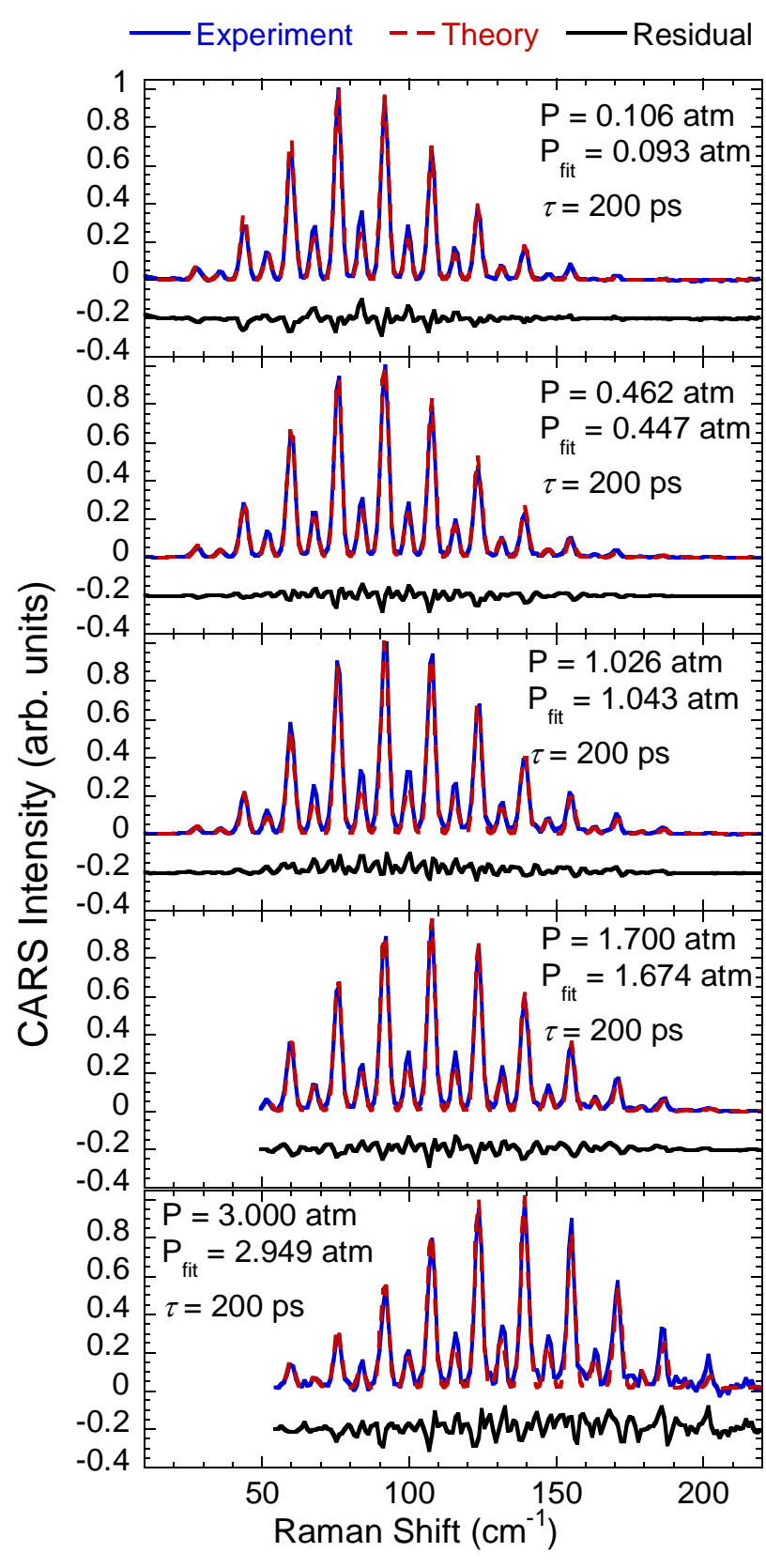

Figure 10. Single-laser-shot spectra for $P=0.1-3.0$ atm for a fixed probe delay of $\tau=200 \mathrm{ps}$. The temperature was fixed at the known value of $T=294$ $K$ in the spectral fitting.

mean single-shot CARS measurements for transducer pressures of $P=0.4-3.03 \mathrm{~atm}$.

A summary of the available single-laser-shot pressure data, at the probe delays of $\tau=100-298$ ps is shown in Table 1. Measurement precision generally improves as $\tau$ is increased, exceeding $2 \%$ by $\tau=297-298$ ps. For $P=1-3$ $\mathrm{atm}$, the accuracy of the single-shot mean pressures ranges from $0.1 \%$ to a worst-case of $6.5 \%$. Accuracy is reduced at $P=0.41 \mathrm{~atm}$, where the results for $\tau=200 \mathrm{ps}$ and $297 \mathrm{ps}$ are $0.04 \mathrm{~atm}$, or $\sim 10 \%$, higher than the transducer
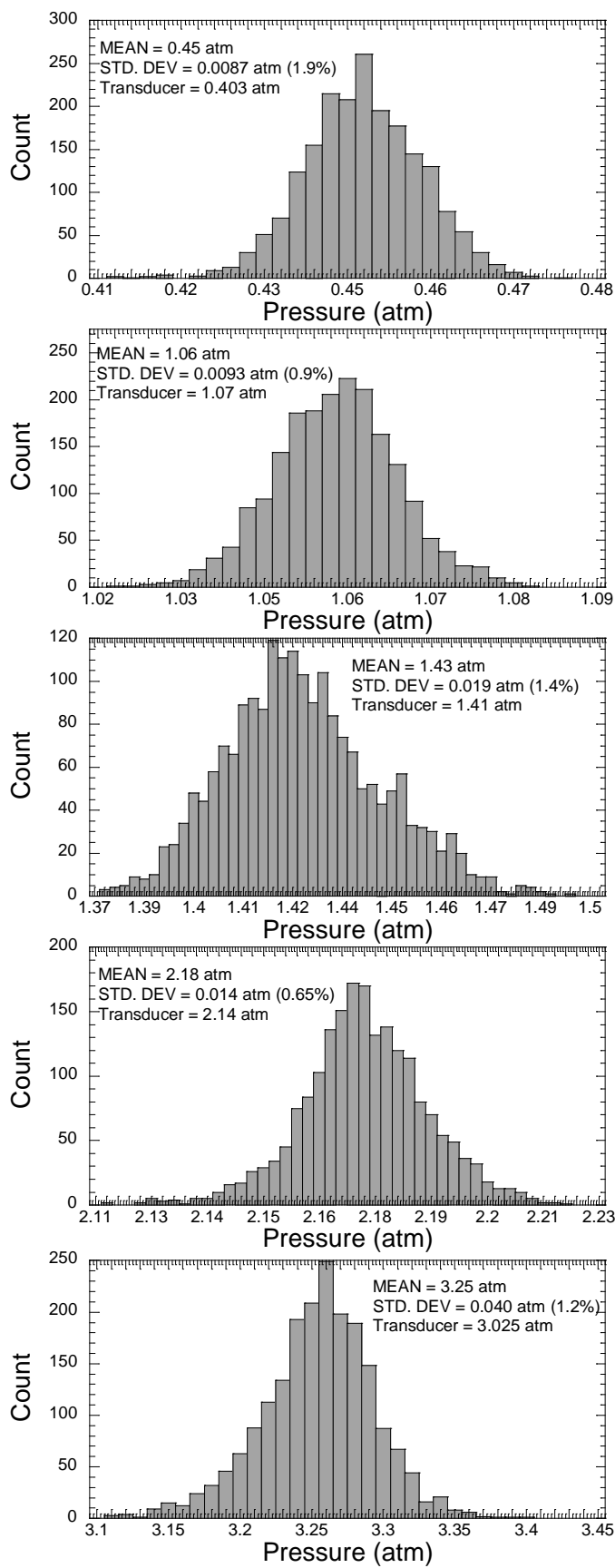

Figure 11. Single-laser-shot histograms of the CARS-measured pressure for gas cell transducer pressure of $P=0.45-3.025$ atm and a fixed probe delay of $\tau=298 \mathrm{ps}$. The temperature was fixed at the known value of $T=294 \mathrm{~K}$ in the pressure fitting. 
pressue, while mean pressures at $\tau=150 \mathrm{ps}$ and $250 \mathrm{ps}$ are $30-40 \%$ higher than the transducer. These results could likely be improved by going to longer probe delays.

\section{Summary and Conclusions}

We have presented a study of the feasibility of pressure monitoring using a hybrid fs/ps rotational CARS diagnostic with a high-energy Second-Harmonic Bandwidth-Compressed (SHBC) picosecond probe beam. Such a system takes advantage of both the temporal and spectral properties of the CARS signal for optimization of the pressure sensitivity. CARS spectra from $\mathrm{N}_{2}$ contained in a room-temperature cell were recorded for pressures ranging from 0.1 to $3.0 \mathrm{~atm}$ and probe-beam delays of $\tau=10-330 \mathrm{ps}$. The pressure scaling of the spectrally integrated decay of the CARS signal was investigated, with time constants within $3 \%$ agreement with model predictions for pressures of $1 \mathrm{~atm}$ and higher. Measured decay constants at pressures of 0.44 and 0.1 atm were 30$50 \%$ lower than predicted, likely as a result of probe-beam walk off at long delays - an effect which could conceivably be calibrated for multi-probe schemes that aim to measure pressure based on the integrated decay.

CARS spectra were observed to be sensitive to pressure for probe delays of $200 \mathrm{ps}$ or more, where the collisional decay of the CARS signal strength places the high probe energies offered by SHBC at a premium. Fits to on-chip integrated spectra provided pressures that were generally within $10 \%$ of the gas-cell transducer when a probe delay of at least 200 ps was employed. The impact of temperature measurement based on a short-delay collision-free CARS spectra - a requirement for pressure monitoring - was systematically assessed. At high-pressures, near $3 \mathrm{~atm}$, the effect of temperature is relatively small, at $2-5 \%$ of the measured pressure. The temperature-induced bias errors increase as the pressure is lowered, reaching $17-27 \%$ at $P=0.44 \mathrm{~atm}$ for a $3 \%$ error in the temperature. In all cases, measurement accuracy is improved by increasing the probe delay, which presents a tradeoff between data fidelity and signal strength. Improved temperature measurements, which have been demonstrated to $\pm 2 \%$ with a similar fs/ps CARS setup [16] will imrove pressure accuracy.

Single-laser-shot spectra were analyzed for pressure at select probe delays with the temperature assumed at the known room-temperature value. The accuracy of the mean single-shot pressures was 0.1 to $6.5 \%$ of the CARSmeasured mean pressures. The precision of the single-shot pressures generally improved as probe delay was increased, reaching levels of $0.65 \%$ to 1.9 percent as $\tau$ approached 300 ps. The observed level of single-shot accuracy (with the correct temperature used in fitting) is comparable to values previously reported using nanosecond CARS in a similar pressure range, while the precision is enhanced by as much as a factor of 5-8 over previous CARS pressure measurements. The results presented here suggest that fs/ps CARS has potential for instantaneous and simultaneous pressure and temperature monitoring based on an arrangement in which at least 2 picosecond probe beams are used.

\section{Acknowledgment}

Sandia is a multiprogram laboratory operated by Sandia Corporation, a Lockheed-Martin Company, for the United States Department of Energy's National Nuclear Security Administration under Contract DE-AC04-94AL85000.

\section{References}

1. Kearney, S. P., Frederickson, K., and Grasser, T. W. "Dual-Pump Coherent Anti-Stokes Raman Scattering Thermometry in a Sooting Turbulent Pool Fire," Proceedings of the Combustion Institute Vol. 32, 2009, pp. 871878.

2. Frederickson, K., Kearney, S. P., Luketa, A., Hewson, J. C., and Grasser, T. W. "Dual-Pump CARS Measurements of Temperature and Oxygen in a Turbulent Methanol-Fueled Pool Fire," Combustion Science and Technology Vol. 182, 2010, pp. 941-959.

3. Beyrau, F., Datta, A., Seeger, T., and Leipertz, A. "Dual-Pump CARS for the Simultaneous Detection of $\mathrm{N}_{2}, \mathrm{O}_{2}$ and $\mathrm{CO}$ in $\mathrm{CH}_{4}$ Flames," Journal of Raman Spectroscopy Vol. 33, 2002, pp. 919-924.

4. Beyrau, F., Seeger, T., Malarski, A., and Leipertz, A. "Determination of Temperatures and Fuel/Air Ratios in an Ethene-Air Flame by Dual-Pump CARS," Journal of Raman Spectroscopy Vol. 34, 2003, pp. 946-951. 
5. Bohlin, A., Nordström, E., Carlsson, H., Bai, X. S., and Bengtsson, P. E. "Pure Rotational CARS Measurements of Temperature and Relative $\mathrm{O}_{2}$ Concentration in a Low-Swirl Turbulent Premixed Flame," Proceedings of the Combustion Institute Vol. 34, No. 2, 2013, pp. 3629-3636.

6. Magnotti, G., Cutler, A. D., and Danehy, P. M. "Development of a Dual-Pump Coherent anti-Stokes Raman Spectroscopy System for Measurements in Supersonic Combustion," Applied Optics Vol. 52, No. 20, 2013, pp. 4779-4791.

7. Foglesong, R. E., Green, S. M., Lucht, R. P., and Dutton, J. C. "Dual-Pump Coherent Anti-Stokes Raman Scattering for Simultaneous Pressure/Temperature Measurement," AIAA Journal Vol. 36, No. 2, 1998, pp. 234240.

8. Woodmansee, M. A., Lucht, R. P., and Dutton, J. C. "Development of High-Resolution Coherent anti-Stokes Raman Scattering for Measuring Pressure, Temperature, and Density in High-Speed Flows," Applied Optics Vol. 39, No. 33, 2000.

9. Kuehner, J. P., Woodmansee, M. A., Lucht, R. P., and Dutton, J. C. "High-Resolution Broadband Coherent antiStokes Raman Spectroscopy: Comparison of Measurements for Conventional and Modeless Broadband Dye Lasers," Applied Optics Vol. 42, No. 33, 2003.

10.Lucht, R. P., Roy, S., Meyer, T. R., and Gord, J. R. "Femtosecond Coherent Anti-Stokes Raman Scattering Measurement of Gas Temperatures from Frequency-Spread Dephasing of the Raman Coherence," Applied Physics Letters Vol. 89, 2006, p. 251112.

11.Roy, S., Kulatilika, W. D., Richardson, D. R., Lucht, R. P., and Gord, J. R. "Gas-Phase Single-Shot Thermometry at $1 \mathrm{kHz}$ Using fs-CARS Spectroscopy," Optics Letters Vol. 34, No. 24, 2009, pp. 3587-3589.

12.Richardson, D. R., Bangar, D., and Lucht, R. P. "Polarization Suppression of the Nonresonant Background in Femtosecond Coherent Anti-Stokes Raman Scattering for Flame Thermometry at $5 \mathrm{kHz}$," Optics Express Vol. 20, No. 19, 2012, pp. 21495-21504.

13. Bohlin, A., and Kliewer, C. J. "Two-dimensional gas-phase coherent anti-Stokes Raman spectroscopy (2DCARS): Simultaneous planar imaging and multiplex spectroscopy in a single laser shot," The Journal of Chemical Physics Vol. 138, 2013, p. 221101.

14.Bohlin, A., Patterson, B. D., and Kliewer, C. J. "Simplified two-beam rotational CARS signal generation demonstrated in 1D," The Journal of Chemical Physics Vol. 138, 2013, p. 081102.

15. Miller, J. D., Dedic, C. E., Roy, S., Gord, J. R., and Meyer, T. R. "Interference-Free Gas-Phase Thermometry at Elevated Pressure Using Hybrid Femtosecond/Picosecond Rotational Coherent Anti-Stokes Raman Scattering," Optics Express Vol. 20, No. 5, 2012, pp. 5003-5010.

16. Kearney, S. P., Scoglietti, D. J., and Kliewer, C. J. "Hybrid femtosecond/picosecond rotational coherent antiStokes Raman scattering temperature and concentration measurements using two different picosecond-duration probes," Optics Express Vol. 21, No. 10, 2013, pp. 12327-12339.

17. Kearney, S. P., and Scoglietti, D. J. "Hybrid femtosecond/picosecond rotational coherent anti-Stokes Raman scattering at flame temperatures using a second-harmonic bandwidth-compressed probe," Optics Letters Vol. 38, No. 6, 2013, pp. 833-835.

18. Kliewer, C. J., Bohlin, A., Nordström, E., Patterson, B. D., Bengtsson, P. E., and Settersten, T. B. "Time-domain measurements of S-branch $\mathrm{N}_{2}-\mathrm{N}_{2}$ Raman linewidths using picosecond pure rotational coherent anti-Stokes Raman spectroscopy," Applied Physics B Vol. 108, 2012, pp. 419-426.

19. Kliewer, C. J., Gao, Y., Seeger, T., Kiefer, J., Patterson, B. D., and Settersten, T. B. "Picosecond Time-Resolved Pure-Rotational Coherent Anti-Stokes Raman Scattering in Sooting Flames," Proceedings of the Combustion Institute Vol. 33, 2011, pp. 831-838.

20. Miller, J. D., Roy, S., Gord, J. R., and Meyer, T. R. "Time-Domain Measurement of High-Pressure $\mathrm{N}_{2}$ and $\mathrm{O}_{2}$ Self-Broadened Linewidths Using Hydrid Femtosecond/Picosecond Coherent Anti-Stokes Raman Scattering," The Journal of Chemical Physics Vol. 135, 2011, p. 201104.

21.Roy, S., Hsu, P. S., Jiang, N., Gord, J. R., Kulatilika, W. D., Stauffer, H. U., and Gord, J. R. "Direct measurements of collisionally broadened Raman linewidths of $\mathrm{CO}_{2}$ S-branch transitions," The Journal of Chemical Physics Vol. 138, 2013, p. 024201.

22.Danehy, P. M., Bathel, B. F., Johansen, C., Cutler, A. D., and Hurley, S. "Spectroscopic measurement techniques for aerospace flows," Von Karman Institute Lecture Series Manuscript. Brussels, Belgium, 2014.

23. Hiller, B., and Hanson, R. K. "Simultaneous planar measurements of velocity and pressure fields in gas flows using laser-induced fluorescence," Applied Optics Vol. 27, 1988, pp. 33-48. 
24.Chang, A. Y., Battles, B. E., and Hanson, R. K. "Simultaneous measurements of velocity, temperature, and pressure using rapid cw wavelength-modulation laser-induced fluorescence of $\mathrm{OH}, "$ Optics Letters Vol. 15, 1990, pp. 706-708.

25.Lachney, E. R., and Clemens, N. T. "PLIF imaging of mean temperature and pressure in a supersonic bluff wake.," Experiments in Fluids Vol. 4, 1998, pp. 354-363.

26. Rothamer, D. A., and Hanson, R. K. "Temperature and pressure imaging using infrared planar laser-induced fluorescence," Applied Optics Vol. 49, 2010, pp. 6436-6447.

27. Miles, R. B., Lempert, W. R., and Forkey, J. N. "Laser Rayleigh scattering," Measurement Science and Technology Vol. 12, 2001, pp. R33-R51.

28. Mielke, A. F., Elam, K. A., and Sung, C. J. "Multiproperty measurements at high sampling rates using Rayleigh scattering," AIAA Journal Vol. 47, No. 4, 2009, pp. 850-862.

29. Fagan, A. F. Personal communication. NASA Glenn Research Center, 2014.

30.Hart, R. C., Herring, G. C., and Balla, R. J. "Pressure measurement in supersonic air flow by differential absorptive laser-induced thermal acoustics," Optics Letters Vol. 32, 2007, pp. 1689-1691.

31. Stauffer, H. U., Miller, J. D., Roy, S., Gord, J. R., and Meyer, T. R. "Hybrid Femtosecond/Picosecond Rotational Coherent Anti-Stokes Raman Scattering Using a Narrowband Time-Asymmetric Probe Pulse," The Journal of Chemical Physics Vol. 136, 2012, p. 111101.

32. Eckbreth, A. C. Laser Diagnostics for Combustion Temperature and Species: Gordon and Breach, 1996.

33. Bohlin, A., Bengtsson, P. E., and Marrocco, M. "On the Sensitivity of rotational $\mathrm{N}_{2}$ CARS Thermometry to the Herman-Wallis Factor," Journal of Raman Spectroscopy Vol. 42, 2011, pp. 1843-1847.

34. Seeger, T., Kiefer, J., Leipertz, A., Patterson, B. D., Kliewer, C. J., and Settersten, T. B. "Picosecond TimeResolved Pure-Rotational Coherent Anti-Stokes Raman Spectroscopy for $\mathrm{N}_{2}$ Thermometry," Optics Letters Vol. 34, No. 23, 2009, pp. 3755-3757.

35.Patterson, B. D., Gao, Y., Seeger, T., and Kliewer, C. J. "Split-probe hybrid femtosecond/picosecond rotational CARS for time-domain measurement of S-branch Raman linewidths within a single laser shot," Optics Letters Vol. 38, No. 22, 2013, pp. 4566-4569.

36. Raoult, F., Boscheron, A. C. I., Husson, D., Sauteret, C., Modena, A., Malka, V., Dorchies, F., and Migus, A. "Efficient Generaton of Narrow-Bandwidth Picosecond Pulses by Freqeuncy Doubling of Femtosecond Chirped Pulses," Optics Letters Vol. 23, No. 14, 1998, pp. 1117-1119.

37. Martinez, O. E. "3000 Times Grating Compressor with Positivie Group Velocity Dispersion: Application to Fiber Compensation in 1.3-1.6 $\mu \mathrm{m}$ Region," Journal of Quantum Electronics Vol. 23, No. 1, 1987, pp. 59-64.

38. Miller, J. D., Slipchenko, M. N., and Meyer, T. R. "Probe-Pulse Optimization for Nonresonant Suppression in Hybrid fs/ps Coherent Anti-Stokes Raman Scattering at High Pressure," Optics Express Vol. 19, No. 14, 2011, pp. 13326-13333. 